\title{
Urban vegetation coverage monitoring technology based on NDVI
}

\author{
Zhu Wenzhe ${ }^{1, a}$, Lei Haochuan ${ }^{1,2, b}$ \\ 1. Department of geological engineering, Qinghai University, Xining city, Qinghai province, China \\ 2. School of information engineering, China university of geosciences, Beijing, China
}

\section{a.3061159300@qq.com \\ b.56242188@qq.com}

Keywords: Urban vegetation coverage; monitoring technology

Abstract: The purpose of this paper is to research with the development of urban and regional change and lead to the change of urban vegetation, for the purpose of ecological environmental quality assessment, urban planning and urban ecological security assessment.

\section{Introduction}

Vegetation coverage is defined as the percentage of the total area of vegetation (including leaves, stems and branches) on the ground ${ }^{1}$. Its change has an important influence on the global energy circulation and the biochemical circulation of matter, and is an important symbol of the environmental change of the regional ecosystem. By obtaining the surface vegetation cover and its change information, it can reveal the law of surface space change, explore the driving factors of change, and analyze and evaluate the regional ecological environment. At the same time, we can provide basic data for subsequent governance, protection and research. Urban vegetation is an important part of urban ecological system, which plays an important role in protecting urban ecological environment. Urban vegetation can effectively alleviate the urban "heat island effect" and improve urban regional climate, etc ${ }^{2}$, . However, due to the development of modern economy, the process of urban development is getting faster and faster. The decrease of urban vegetation cover caused by the process of rapid urbanization and the short-term and long-term effects of urbanization on urban ecosystem have become the focus of attention. Therefore, more and more scholars in the academic circle have carried out a series of researches on urban vegetation and vegetation coverage.

Before the application of remote sensing technology, the monitoring of vegetation coverage in large areas can only be completed by field investigation, which must consume a large amount of manpower and material resources. At the same time, it is inevitable that there are many subjective and objective uncertainties in the field investigation, which will affect the accuracy of the final result $^{4}$. Remote sensing technology can continuously provide digital images with four dimensional (three-dimensional space and time) characteristics, so remote sensing images can be used to accurately record the landscape characteristics of vegetation coverage, and the vegetation index information can be extracted accurately, quickly and continuously, providing a reliable guarantee for dynamic coverage of vegetation coverage ${ }^{5}$. The results show that using remote sensing data to estimate urban vegetation coverage and spatial distribution and dynamic analysis has become an important method to monitor urban ecological environment ${ }^{6}$.

Xining is located in the east of Qinghai, the Yellow River tributary of Huangshui river, the highest altitude of 4,394 meters in the city of Xining, the urban center is 2,775 meters above sea level. Xining is a semi-arid climate of the continental plateau, with little rain, large evaporation and 
extremely fragile ecological environment. At the same time, Xining is the only city with a population of over 1 million in the Qinghai-Tibet plateau. In recent years, with the continuous development of economy, the expansion of population size and the expansion of cities, a large number of urban problems have also been brought. Therefore, it is of practical significance to monitor the vegetation coverage of Xining city. In this paper, the ETM+ remote sensing data of 2006 and 2016 are used in the study area of Xining region. Using the normalized vegetation index (NDVI), the method is used to estimate the vegetation coverage. Finally, the vegetation coverage of these two periods is calculated. The purpose of this paper is to research with the development of urban and regional change and lead to the change of urban vegetation, for the purpose of ecological environmental quality assessment, urban planning and urban ecological security assessment.

\section{Research area}

Xining is located in the eastern edge of Qinghai-Tibet plateau, the high terrain northwest, southeast low, upstream of Huangshui river, east longitude $101^{\circ} 49^{\prime} 17$ ", latitude $36^{\circ} 34^{\prime} 13$ ". The average urban area is 2295 meters above sea level, which is a semi-arid climate of the continental plateau with an average temperature of 6.1 degrees Celsius. Xining is the provincial capital of Qinghai province, and the political, economic, cultural, education, technology, transportation and trade center of the province. It has a long history of more than 2,100 years. Xining city includes the east, the center, the west, the north of the city and the Datong, Huangzhong, Huangyuan and the national Xining economic development zone and the city south new area.The total area is 7665 square kilometers.

\section{Data processing and technical route}

\subsection{The data source}

This study took advantage of the Landsat7 ETM+ remote sensing data of the two phases in 2006 and 2016 in Xining city, with a spatial resolution of $30 \mathrm{~m} * 30 \mathrm{~m}$. In addition to the remote sensing data, it also includes the topographic map of 1: 500, 000 in Xining city, and 1: 4 million vector drawings in the county boundary. In order to extract vegetation information more reasonably and efficiently, remote sensing images of different years of the same month are usually selected. The remote sensing images we selected were in July in the lush vegetation of Xining. The two images are basically cloudless, and the imaging quality is better. So that changes in vegetation in the study area is comparable.

\subsection{Pre-processing of remote sensing images}

\subsubsection{Geometric correction}

In order to carry out dynamic comparison and dynamic change of area size, it is necessary to carry out geometric correction and two-phase image registration for remote sensing images. Xining 1:500 million topographic map was used as standard reference data. Using UTM projection, WGS84 coordinate system, 32 control points were selected, and the control points were calculated with cubic polynomial. Obtaining the remote sensing image in 2006 by using a double - linear interpolation method. By using the image method, the remote sensing image of 2016 was matched to the remote sensing image in 2006. After the 28 control points were selected, three polynomial adjustment calculations and bilinear interpolation methods were performed for the 28 control points, and the remote sensing image of 2016 was obtained through geometric correction. The maximum error of geometric correction is not more than 0.5 pixels. The spatial position registration of pixels in the same position is guaranteed.

\subsubsection{Atmospheric correction}

According to previous research results and experience, when using NDVI to calculate the 
vegetation coverage in the study area, if atmospheric correction is not carried out, the calculation error of NDVI value in local area will be more than $50 \%^{7}{ }^{8}$. In this paper, using the FLAASH atmospheric correction module in ENVI5.1 and the k-t aerosol inversion model. After processing, we got the spectral curve of the atmospheric correction result image at the same pixel point (FIG. 1 is the spectral curve of vegetation before atmospheric correction, and FIG. 2 is the spectral curve of vegetation after atmospheric correction). By comparing the spectral curves of the same pixel points on the two images, the corrected vegetation spectral curve is closer to the true vegetation spectrum.

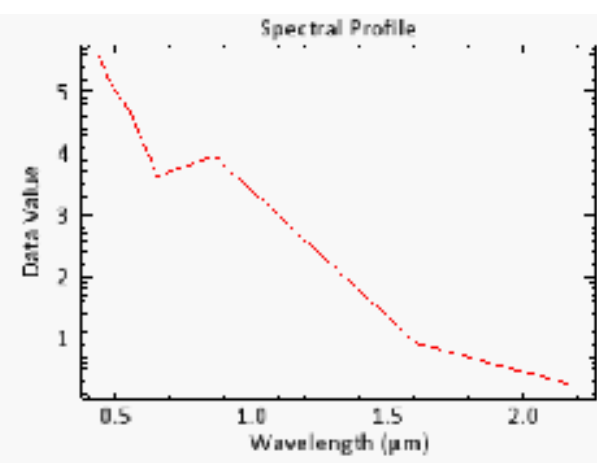

FIG. 1

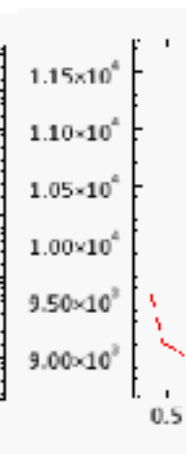

0.5

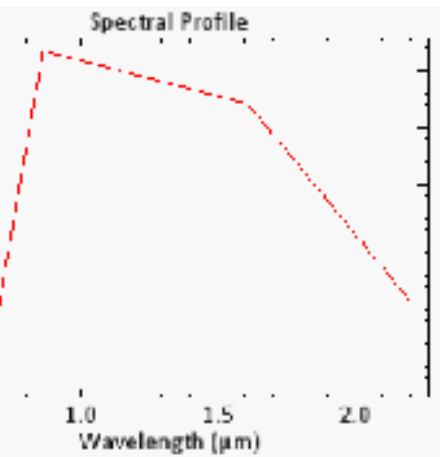

FIG. 2

\subsubsection{Image Mosaic and cropping}

In the first place, the two images were combined together into a wide and seamless remote sensing image by using Seamless Mosaic in ENVI5.1. Then, with the support of arcgis10.2, the vector map of the city is extracted by using the vector map of the national 1: 4 million counties. With the support of ENVI5.1, remote sensing image cutting is carried out by using the vector map of the city. In the end, we obtained the remote sensing images consistent with the research area.

\section{The vegetation coverage of the inverse model based on NDVI}

\subsection{Normalized vegetation index NDVI}

The experiment shows that NDVI is sensitive to the change of soil background. It is a comprehensive reflection of vegetation type, cover morphology and growth condition in a pixel. Its value depends on the vegetation coverage and leaf area index. NDVI has a wide detection range of vegetation coverage, and has better time and space adaptability. Therefore, it is widely used ${ }^{9}$. It mainly has the following advantages:

(1) The sensitivity of vegetation detection is higher

(2) The coverage of vegetation coverage is wide

(3) Eliminate the shadow and radiation interference of topography and community structure

(4) Reduce the noise caused by the high Angle of the sun and the atmosphere

Therefore, NDVI was selected in this study to monitor vegetation changes.

In this study, two years of NDVI were calculated based on ETM images in 2006 and ETM images in 2016. We obtained the NDVI gray scale image of Xining city in 2006 and 2016 (FIG. 3、 FIG. 4). The brighter the brightness on the image, the higher the vegetation coverage. The lower the brightness, the lower the vegetation coverage. 


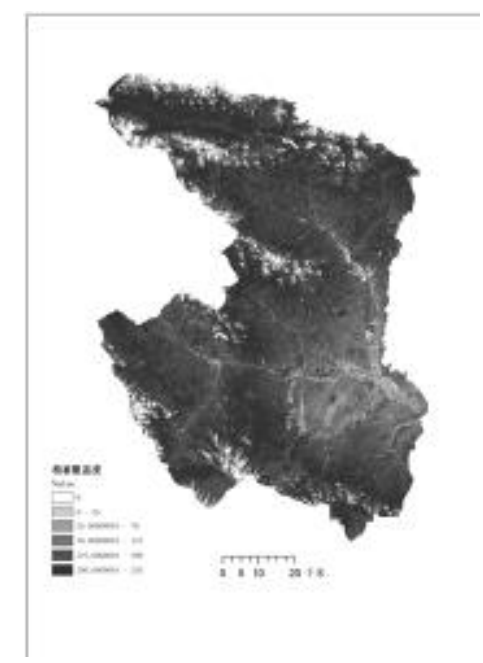

FIG. 3 NDVI gray scale of Xining city in 2006

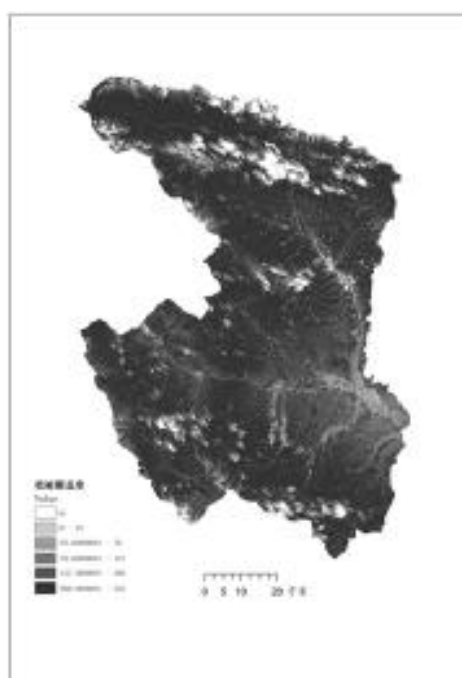

FIG. 4 NDVI gray scale of Xining city in 2016

\subsection{Pixel model ${ }^{10}$}

The principle of the meta-binary model is to assume that the reflectivity $\mathbf{R}$ of a pixel in the image can be divided into two parts, the reflectance of pure vegetation $R_{v}$ and non-vegetated parts of the pure vegetation $R_{f}$. The reflectivity value of any pixel can be expressed as a linear weighted sum of vegetation cover and non-vegetation coverage:

$$
\mathrm{R}=R_{v}+R_{f}
$$

Suppose that the ratio of an area covered by vegetation in the image is $f_{c}$. This is the vegetation coverage of pixels. So the proportion of non-vegetated area is $1-f_{\sigma}$. If the pixel is covered by vegetation, the reflectivity is $R_{v \in g}$. If the pixel is not covered by vegetation, the reflectivity is $R_{s o i l}$. Therefore, the information provided by the vegetation section of the mixed pixel is $R_{v}$. It can be expressed as the product of pure vegetation reflectivity $\left(\boldsymbol{R}_{\text {veg }}\right)$ and vegetation cover area in pixels $\left(f_{e}\right)$.( Formula (2)).Information contributed by non-vegetative components $R_{f}$ can be expressed as the product of $R_{s o i l}$ and $1-f_{a}$. (Formula (3)):

$$
\begin{aligned}
& R_{v}=f_{o} * R_{v \boxminus g} \\
& R_{f}=\left(1-f_{o}\right): R_{\text {goiI }}
\end{aligned}
$$

By calculating formula (1), formula (2) and formula (3), we can get a formula for calculating vegetation coverage. The following: 


$$
f_{o}=\left(R-R_{\text {soil }}\right) /\left(R_{\text {veg }}-R_{\text {soil }}\right){ }^{11}
$$

\subsection{Using the normalized vegetation index to estimate vegetation coverage}

\subsubsection{Vegetation coverage formula}

According to the principle of the meta-binary model, we can represent the NDVI value of a pixel as a form of surface composition of vegetation cover and non-vegetation cover. The formula for calculating vegetation coverage can be expressed as ${ }^{12}$ :

$$
f_{e}=\frac{N D V I-N D V I_{\text {sDil }}}{N D V I_{\text {VEg }}-N D V I_{\text {gin }}}
$$

$N D V I_{\text {soil }}$ is the NDVI value of completely bare soil or no vegetation cover area. $N D V I_{\text {ves }}$ represents the NDVI value that is completely covered by vegetation. In actual work, due to the lack of large area surface measured data for reference, NDVI statistical histogram was given a confidence interval. The NDVI value of the whole vegetation cover and the whole soil was determined by the maximum and minimum values in the region ${ }^{13}$. In ENVI5.1 software, according to the NDVI statistical histogram of 2006 and 2016, the cumulative percentage of the cumulative percentage is $2 \%$ and $95 \%$ confidence interval. The cumulative percentage $<2 \%$ is approximately pure soil coverage, while $>95 \%$ is covered by whole vegetation, and its corresponding NDVI value is the NDVI value of the pixel of pure soil cover and full vegetation coverage. After calculation, the parameters of 2006 are: $\quad N D V I_{s o n}=0.0314 \quad, \quad N D V I_{v e g}=0.6153 \quad ; \quad 2016$ parameters: $N D V I_{\text {soiI }}=0.0758, N D V I_{v e g}=0.6879$.

\subsubsection{Figure out the vegetation coverage and grade}

Using the formula (5) in ENVI5.1 software, the band was calculated. The vegetation coverage was quantified as the transformation model. Then the vegetation coverage in the study area was divided into five grades according to the actual vegetation coverage. $f_{c}<10 \%$ 、 $10 \%<=f_{c}<30 \%, 30 \%<=f_{c}<50 \%, 50 \% \leqslant=f_{c}<70 \%, f_{e}>=70 \%\left(f_{c}\right.$ is based on the vegetation coverage of pixels). According to the degree of vegetation coverage, the research area was divided into the following areas: inferior vegetation coverage, poor vegetation coverage, medium vegetation coverage, good vegetation coverage, excellent vegetation coverage. We then obtained two levels of vegetation coverage at different times (FIG. 6, FIG. 7). 


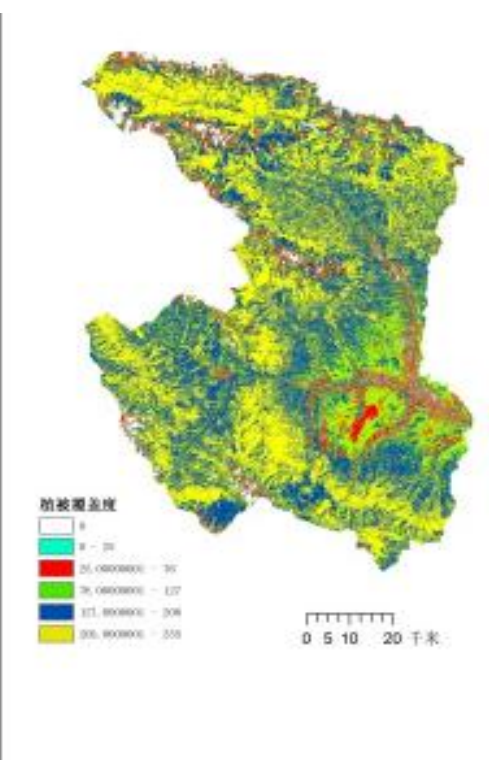

FIG. 5 Vegetation coverage level map in 2006

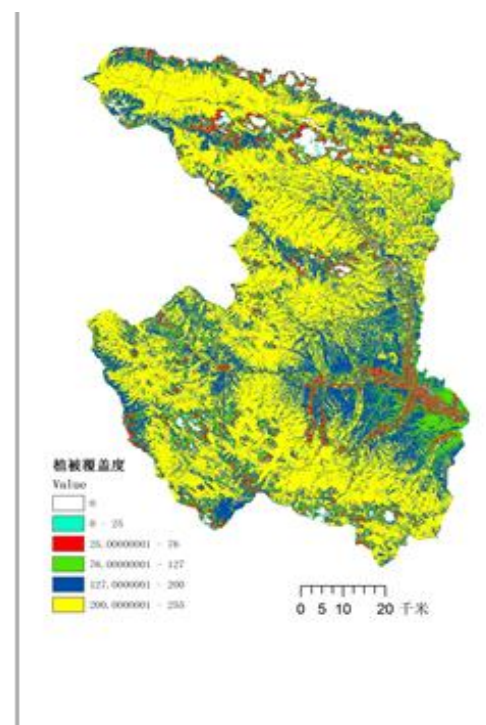

FIG. 6 Vegetation coverage level map in 2016

\subsubsection{Calculate the area of each grade according to the vegetation coverage level map}

Through the Statistics Results in ENVI5.1, the number of pixels contained in the vegetation coverage level map (FIG. 6 and 7) was calculated, and the specific area was calculated. The specific area changes of each vegetation cover level are shown in table 1.

Table 1 The change of vegetation coverage of different grades in Xining city, Qinghai province from 2006 to 2016

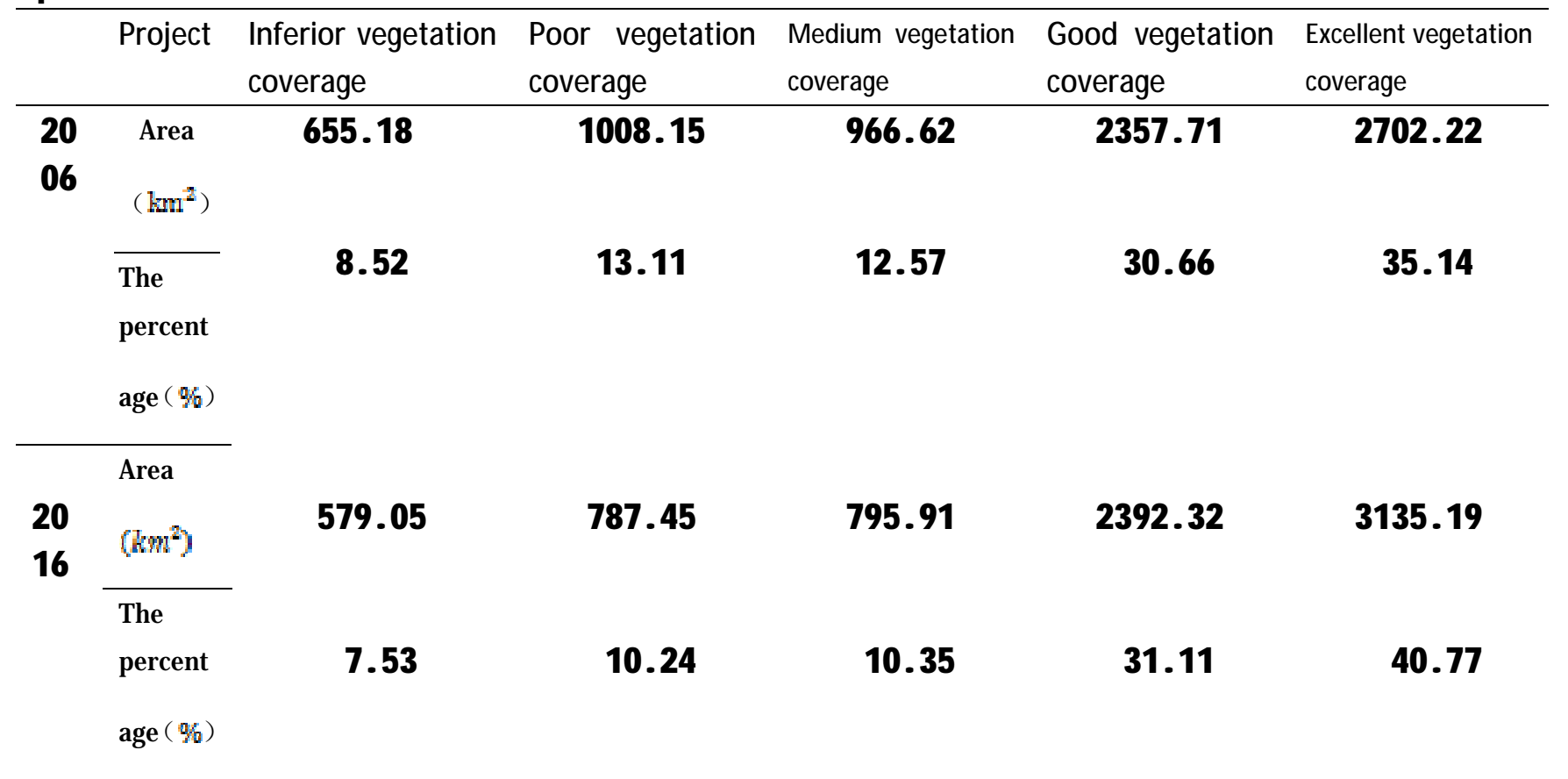


Area increase and

\begin{tabular}{lccccc}
$\begin{array}{l}\text { decrease } \quad \text { from } \\
2006 \text { to } 2016 .\end{array}$ & -76.13 & -220.70 & 34.61 & 432.97 \\
$\left(\mathrm{~km}^{2}\right)$ & & & & & \\
\hline $\begin{array}{l}\text { Rate of change } \\
\text { from } 2006 \text { to } 2016\end{array}$ & -11.62 & -21.89 & -17.66 & 1.47 & 16.02 \\
$(\%)$ &
\end{tabular}

\section{Results and analysis}

\subsection{Spatial distribution and overall change characteristics of vegetation coverage in Xining city}

5.1.1 The dynamic change characteristics of the spatial distribution of vegetation coverage in Xining city

Through the analysis of Xining city from 2006 to 2016 different levels of vegetation coverage area of change, it is easy to know: By comparing the vegetation coverage in 2006 and 2016, inferior vegetation coverage, poor vegetation coverage and medium vegetation coverage area decreased significantly. Good vegetation coverage and excellent vegetation coverage have significantly increased. The area of the inferior vegetation coverage and poor vegetation coverage and medium vegetation coverage area were decreased by $76.13 \mathrm{~km}^{2}, 220.70 \mathrm{~km}^{2}, 170.71 \mathrm{~km}^{2}$. The rate of decrease of inferior vegetation coverage, poor vegetation cover and medium vegetation coverage was also quite fast, $11.62 \%, 21.89 \%, 17.66 \%$ respectively. The good vegetation coverage and excellent vegetation coverage area are increased, the area of the two respectively increased by $34.61 \mathrm{~km}^{2}$ and $432.97 \mathrm{~km}^{2}$. In terms of the change speed, the growth rate of excellent vegetation coverage area was the largest, with a growth rate of $16.02 \%$. The growth rate of good vegetation coverage was the smallest, only

\section{$1.47 \%$.}

\subsubsection{Reasons for the overall change of vegetation cover in Xining city}

Through table 1 data and table 1 data analysis, it can be concluded that the vegetation coverage of Xining city from 2006 to 2016 has been developing well, and the ecological environment has continued to improve. The rate of decrease of inferior vegetation coverage and poor vegetation coverage was also quite obvious, $11.62 \%$ and $21.89 \%$ respectively. The growth rate of excellent vegetation coverage area was rapid, with a growth rate of $16.02 \%$. The main reasons for the overall change of vegetation cover in Xining are as follows : (1) Natural factors: In recent years, the temperature in Xining city has increased, the precipitation has increased, the climate has been transformed from warm and dry to warm and humid, which has promoted the growth of vegetation, increased the survival rate of saplings, and reduced the difficulty of afforesting barren hills. (2) Macro policy: Xining city aims to create a national forest city. In recent years, based on the natural 
ecological characteristics of urban areas, the forestry ecological project has been vigorously implemented. These projects have achieved double growth in the quantity and quality of forest resources. There are 203 voluntary tree planting bases and 95.42 percent of the duty rate. According to the statistical results in the yearbook of Xining, the city of Xining completed the key project of afforestation of 1105,000 mu and 63.64 million mu of forest cover in 2006 2016, and planned to make full efforts to build the national eco-park of the north and south mountain.

\subsection{Change of vegetation coverage in each administrative area}

For the convenience of the vegetation coverage of each administrative region in Xining city comparison, statistics, analysis, with the support of ENVI software, we added the vegetation coverage data of Xining city and the border map of Xining city, get the vegetation coverage map in each county, and calculate the average vegetation coverage of each district. The statistical results are shown in table 2.

Table 2 Vegetation coverage and changes in each administrative area

\begin{tabular}{lcccccccccc}
\hline Project & \multicolumn{2}{c}{$\begin{array}{c}\text { Inferior vegetation } \\
\text { coverage }\end{array}$} & \multicolumn{2}{c}{$\begin{array}{c}\text { Poor vegetation } \\
\text { coverage }\end{array}$} & \multicolumn{2}{c}{$\begin{array}{c}\text { Medium vegetation } \\
\text { coverage }\end{array}$} & $\begin{array}{c}\text { Good vegetation } \\
\text { coverage }\end{array}$ & $\begin{array}{c}\text { Excellent vegetation } \\
\text { coverage }\end{array}$ \\
\hline $\begin{array}{c}\text { The Center of } \\
\text { Xining city }\end{array}$ & 2006 & 2016 & 2006 & 2016 & 2006 & 2016 & 2006 & 2016 & 2006 & 2016 \\
Huangyuan & 14.43 & 4.23 & 24.73 & 20.28 & 44.61 & 15.29 & 17.62 & 45.66 & 7.33 & 14.54 \\
Huangzhong & 25.59 & 10.32 & 18.63 & 17.31 & 13.27 & 12.38 & 36.34 & 36.17 & 17.33 & 19.82 \\
Datong & 8.37 & 10.76 & 10.86 & 22.35 & 19.44 & 14.50 & 30.56 & 20.17 & 30.77 & 32.22 \\
\hline
\end{tabular}

In the past ten years, the vegetation coverage has not changed much in Xining central city and Huangyuan county. The change is more obvious in Datong county and Huangzhong county. Among them, the proportion of area of the poor vegetation coverage in Datong county increased from $10.86 \%$ to $22.35 \%$. This is mainly due to the low level of social and economic development in Datong county, which was designated as a national poverty-stricken county in 2002 . In order to achieve economic development, a lot of exploitation of all kinds of natural resources resulted in grassland area was reduced, and the quality is not high, pasture degradation trend is obvious. People have weak awareness of environmental protection. Livestock farming is lagging behind. Farmers and herdsmen increase the number of livestock and overgrazing to increase their personal income through traditional feeding methods, which has a negative impact on the restoration of grassland ecosystem. A large number of migrant workers excavated cordyceps sinensis and Tibetan medicine, which caused the destruction of surface vegetation and threatened the land ecological security of Datong county.

The protection function of forest ecosystem is weakened. In the early stage of social and economic development, Datong county lagged behind in the construction of ecological shelterbelt. Natural forest are mainly distributed in the whole county of Beichuan river tributary, the east of Dongxia river and empress mountain area. The whole county's natural forests are scattered and discontinuous, so there are many problems such as wide area, less personnel, less capital and more difficulty.

In Huangzhong county, the inferior vegetation coverage and poor vegetation coverage area have been greatly reduced and the benign transformation has been achieved. This is mainly due to the 
great efforts of Xining city in 2006 2016 to promote the conversion of farmland to forest and grassland. Huangzhong county is one of the important counties in Xining to return farmland to forest and grassland. From the data, it can be seen that the proportion of the inferior vegetation coverage and poor vegetation coverage in Huangzhong county decreased from $25.59 \%, 19.12 \%$ to $10.33 \%, 9.87 \%$ respectively. It can be seen that the conversion of farmland to forest and grassland has obviously improved the vegetation coverage level in the hilly area and effectively promoted the improvement of vegetation.

\section{6 conclusions}

Through estimation and comparative analysis of vegetation coverage in different periods, we can better reflect the current status and dynamics of vegetation in the study area. And it reveals the process of urbanization to the urban vegetation. We can evaluate the dynamic change of urban vegetation coverage in a more comprehensive and scientific way. In the next step of scientific research, the dynamic change of urban vegetation coverage and its driving mechanism can be studied in multi-temporal data.

$\left[{ }^{1}\right]$ Leprieu C,Kerr Y H,Mastorchio S, et al.Monitoring Vegetation Cover Across Semi-arid Regions, Comparison of Remote Observations from Various Scales [J].International Journal of RemoteSensing,2010,21(2):281-300

$\left.{ }^{2}\right]$ Chen Tao, Li Pingxiang, Zhang Liangpei, 1998 2002 dynamic analysis of vegetation coverage in Wuhan area, $2008.05(06)$

[3] Ding Guodong, Study on the knowledge and grading standards of vegetation in the evaluation of desertification in district cities -- a case study of maousu district. [J] Journal of soil and water conservation 2014.18(1):159-160

$\left[{ }^{4}\right]$ Qin Wei, Research progress of vegetation coverage and measurement method. [J]. Journal of northwest agricultural and forestry university (natural science edition).2006.34 (9):163-166

$\left[{ }^{5}\right]$ Chen shupeng, Tong qingxi, Guo huadong. Research on the mechanism of remote sensing information. [M]. Science press, 1998.345349.

$\left[{ }^{6}\right]$ Rundquist B C.The Influence of Canopy Green Vegetation Fraction on Spectral Measurements over Native Tallgrass Prairie[J].Remote Sensing of Environment,2002.81(1) 1129-135.

$\left[{ }^{7}\right]$ Shen tao, Yuan Chunqiong, Liu Yuan. Remote sensing study on the relationship between the intensity distribution and vegetation cover of the heat island in urumqi. [J]. Xinjiang meteorological,2004,27(1):28-30

$\left[{ }^{8}\right]$ Zhao Yingshi, Principle and method of remote sensing application analysis. [M] , Beijing science publishing du., $2003.387-398$.

$\left[{ }^{9}\right]$ Mo Yao, Zheng Youfei, Chen Huailiang.. Analysis of vegetation cover changes in huang-huaihai area from 1982 to 2000. [J]. Remote sensing technology and application.,2007,22(3):397-398.

$\left[{ }^{10}\right]$ Zribi M,Le Hegarat-Masde,Derivation of Wild Vegetation Cover Density in Semi-arid Region: ERS2/SAR Evaluation [J].Intermational Journal of Remote Sensing,2013,(24), 1335-1352.

$\left[{ }^{11}\right]$ Chen jin, Chen yunxiang, He chunyang, The subpixel model and application of vegetation coverage based on land cover classification. [J]. Journal of remote sensing,2011,5(6).416-423.

$\left[{ }^{12}\right]$ Gao Fei, Xing Wenyuan Remote sensing monitoring and analysis of grassland cover in hejing county. [J]. Pratacultural science ,2007.24(4).27 30.

$\left[{ }^{13}\right]$ Chen Tao, Li Pingxiang, Zhang Liangpei, 1998 2002 dynamic analysis of vegetation coverage in Wuhan area, $2008.05(06)$ 